\title{
PROPOSTA MULTIMETODOLÓGICA DE ENSINO PARA A DISCIPLINA DE DESIGN DE PLACAS DE CIRCUITO IMPRESSO
}

João Pedro Aguiardos Santos - jpedroags@gmail.com*

João Inácio Yutaka Ota-yutaka@dsce.fee.unicamp.br*

Universidade Estadual de Campinas (Unicamp), Faculdade de Engenharia Elétrica e Computação (FEEC) *

Av. Albert Einstein, 400

Cidade Universitária "Zeferino Vaz"

13083-970-Campinas - SP*

Resumo: O artigo apresenta uma proposta multimetodológica de enfrentamento à resistência dos discentes expostos às metodologias ativas aplicadas em uma disciplina eletiva voltada ao estudo, análise e projeto de Placas de Circuitos Impressos (PCIs) dentro de um curso de Engenharia Elétrica. A importância de formar profissionais autônomos em seu aperfeiçoamento, confortáveis no trabalho em equipe e capazes de interagir e discutir com clientes e colegas aspectos complexos ligados ao projeto de PCIs demanda o desenvolvimento de tais habilidades desde a etapa de formação do profissional. Metodologias ativas têm sido empregadas, em detrimento do método comportamental tradicional, na missão de ajudar os alunos a ir além da simples retenção de informação. A aprendizagem baseada em projetos $(A B P)$ é uma excelente alternativa para a disciplina proposta. Porém, problemas de relações interpessoais e a dificuldade com a aprendizagem autodidata são algumas das principais barreiras à implementação efetiva da ABP. Dentro dos aspectos de Alinhamento Construtivo e da Taxonomia de Bloom, entende-se que metodologias que envolvam uma aproximação da relação entre aluno e professor e uma construção gradual e hierárquica de capacidades ao longo de um periodo criam as bases para os alunos desenvolverem tais habilidades. Propõese e detalha-se, assim, um programa disciplinar dividido em três etapas, nas quais métodos de avaliação distintos procuram facilitar a adaptação e aumentar o engajamento dos discentes de acordo com os objetivos educacionais esperados. $O$ aspecto social e construtivo da proposta multimetodológica permite sua aplicação em qualquer disciplina que tenha a realização e análise de projetos em seu escopo.

Palavras-chave: Alinhamento Construtivo. Compatibilidade Eletromagnética. Design de Placas de Circuito Impresso. Educação em Engenharia. Taxonomia de Bloom.

\section{INTRODUÇÃO}

De acordo com Zepke e Leach (2010), não é uma tarefa fácil para educadores e professores garantir que o aluno seja capaz de abstrair, estender e relacionar o conhecimento com responsabilidade e autonomia ao mesmo tempo que promova o desenvolvimento social, cultural e torne os alunos em cidadãos ativos e agentes de transformações na sociedade. Para tanto, o professor precisa avaliar-se e se adaptar às mudanças tecnológicas e sociais constantemente, entendendo seu papel como o agente responsável pela transformação do indivíduo (BRYSON e HAND, 2007). O ensino e a aprendizagem precisam ser encarados e encaminhados com rigor e argumentação, e não baseados em "achismos" ou "verdades". A partir dessa postura, o docente descobre que existem inúmeras metodologias e teorias educacionais, que oferecem ferramentas e apresentam resultados significativos (LEFRANCOIS, 2019). 
Tennant et al. (2009), destaca que cabe ao docente escolher os pontos positivos de cada método, de acordo com sua visão de ensino, perfil dos alunos, infraestrutura e habilidades que se busca desenvolver nos alunos. Por fim, esta não é uma tarefa simples, e envolve a participação e atuação em diferentes esferas da sociedade, as quais tornam-se realidade a partir de: (i) políticas educacionais que incentivem as instituições de ensino a utilizar novas metodologias de ensino, (ii) departamentos que repensem a forma de engajar o professor, (iii) universidades que ofereçam formação continuada e sólida e (iv) avaliações institucionais coerentes (PRETTO e RICCIO, 2010; BEHRENS, 2007; MENEGHEL et al., 2006).

De acordo com Guedes e Santos (2019), alunos expostos a metodologias ativas de ensino e aprendizagem precisam desenvolver habilidades muito além das promovidas pelo método tradicional comportamental, tais como a aprendizagem autodidata, a capacidade de trabalhar em equipe, a capacidade de resolver problemas lógicos, entre outros. Porém, segundo Prince e Felder (2007) a exigência de aquisição dessas habilidades aumenta a resistência dos alunos à aplicação do método, sendo os problemas interpessoais que ocorrem ao trabalhar em grupo e a necessidade de aprendizagem autodidata algumas das principais dificuldades percebidas pelo docente ou levantadas pelos alunos. Assim, caso o docente não esteja preparado, esses problemas podem diminuir o engajamento dos alunos e consequentemente o aprendizado.

A partir dessa perspectiva, é apresentado uma proposta multimetodológica para uma disciplina de desenvolvimento de placas de circuito impresso, na qual são abordados uma série de tópicos: (i) boas técnicas de design para reduzir e evitar problemas de compatibilidade eletromagnética, (ii) tecnologias de fabricação, planejamento, montagem e normas de certificação, (iii) capacitar o aluno a projetar, avaliar e analisar placas de circuito impresso e (iv) relacionar e integrar os conceitos estudados durante outras disciplinas do curso de graduação. A proposta multimetodológica baseia-se na mescla de três metodologias: (i) expositiva e tradicional, (ii) ativa baseada em estudo de casos e (iii) ativa baseada em projetos.

\section{FUNDAMENTAÇÃO TEÓRICA PARA PROPOSIÇÃO DA DISCIPLINA}

\subsection{Alinhamento construtivo}

Segundo Biggs (1996), os docentes precisam ser claros quanto ao conhecimento que é necessário para que se demonstre um certo nível esperado ou desejável de desempenho. Assim, o alinhamento construtivo é uma forma de planejamento de ensino que está relacionado com a construção de um portifólio para avaliar se os alunos atingem ou não os objetivos estabelecidos no processo de aprendizado. Ao deixar claro aos alunos essa relação, eles podem refletir sobre o que eles aprenderão nas aulas e como farão para alcançar esses objetivos, e é papel do professor passar atividades de ensino e aprendizagem que auxilie os alunos a alcançar os objetivos, conforme descrito na Figura 1 (BIGGS, 2011). Portanto, segundo Souza (2016), o professor deve planejar a aula pensando no que ele quer que os alunos sejam capazes de fazer e em seguida avaliar se os alunos de fato aprenderam, evidenciando que o processo se relaciona com os objetivos estabelecidos e resultados esperados.

\subsection{Taxonomia de Bloom}

Para Ferraz e Belhot (2010), o planejamento da disciplina é importantíssimo, pois, sem um plano que delimite o conteúdo e estratégias educacionais eficientes, o docente pode enfrentar altos níveis de evasão nas disciplinas e até mesmo ansiedade pessoal por observar que seus alunos não estão atingindo o grau de habilidade e competência esperada. Ainda segundo Ferraz e Belhot (2010), isso pode ocorrer devido à dificuldade do docente de compreender a importância do conteúdo ministrado e não alinhar os objetivos da disciplina com as avaliações que são realizadas, acarretando em resultados de aprendizagem diferentes daquilo que o docente esperava. 


\section{Evento On-line}

Figura 1 - Alinhamento construtivo.

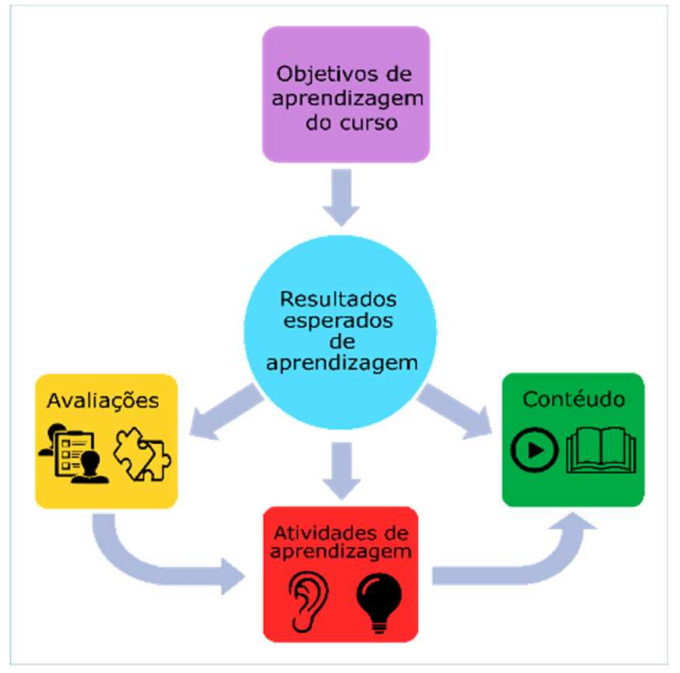

Fonte: Confeccionado pelo autor.

A Taxonomia de Bloom (BLOOM et al., 1956) é uma ferramenta para auxiliar o docente na classificação hierárquica dos objetivos de aprendizagem. Conforme apresentado na Figura 2, classifica-se os objetivos de aprendizagem a partir do nível mais simples para o nível mais complexo, de forma gradual (KRATHWOHL e ANDERSON, 2009). Trata-se de um instrumento que permite a criação de estratégias diferenciadas e facilitadoras no processo de aprendizagem dos alunos, tornando as avaliações mais justas e estimulando o desempenho dos alunos. Deve-se notar, porém, que a estrutura hierárquica da Figura 2 não implica que níveis altos de aprendizagem somente são atingidos quando os níveis inferiores estão superados (GARAVALIA, 1999). Tal flexibilidade é reforçada pela observação de que certos estímulos provenientes de níveis mais complexos podem facilitar o entendimento de conteúdos didáticos de níveis inferiores (FERRAZ e BELHOT, 2010).

Figura 2 - Taxonomia de Bloom

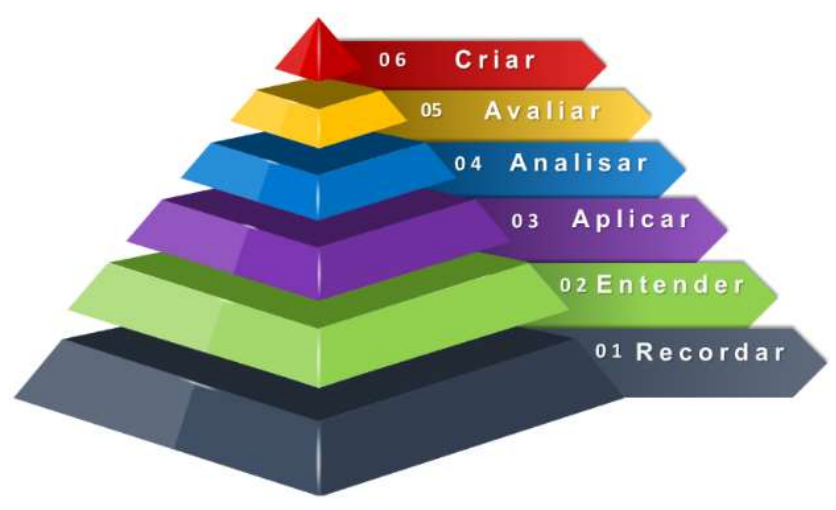

Fonte: Confeccionado pelo autor.

\subsection{Método Trezentos}

Apresentado por Fragelli e Fragelli (2017), esse é um método colaborativo que separa os alunos em grupos de acordo com o rendimento nas avaliações. Alunos com bom rendimento são chamados de "ajudantes" e os com rendimento não satisfatório são chamados de "ajudados". Assim, formam-se grupos compostos por alunos ajudantes e ajudados. Os alunos 
ajudados têm o direito de refazer avaliações enquanto que o papel dos ajudantes é auxiliar os ajudados a alcançar melhores notas nas novas avaliações. Os ajudantes não refazem as avaliações, mas sua nota também é melhorada de acordo com a melhora de nota alcançada pelos ajudados e com o grau de ajuda dada no grupo, o qual é medido através de um questionário com escala Likert de cinco pontos preenchido tanto pelo aluno ajudante quanto pelo aluno ajudado, variando de 1 (ajudei/ajudou nada) para 5 (ajudei/ajudou bastante).

\section{PROJETO DE PLACAS DE CIRCUITO IMPRESSO}

Segundo Paul (2006), campos eletromagnéticos produzidos por um circuito eletrônico podem resultar em interferências no funcionamento de circuitos eletroeletrônicos. Deste modo, um equipamento é dito compativel eletromagneticamente quando tais interferências não provocam o mau funcionamento de equipamentos externos ou em partes internas de si mesmo. Existem normas nacionais e internacionais definidas por órgãos como a CISPR (do francês Comité International Spécial des Perturbations Radioélectriques), o IEC (do inglês International Electrotechnical Commission) e o Instituto Nacional de Metrologia, Qualidade e Tecnologia (Inmetro) que definem níveis máximos de interferência conduzida e irradiada que um equipamento pode produzir e requisitos mínimos de imunidade para que um dado dispositivo funcione perfeitamente em qualquer ambiente eletromagnético.

Assim, é fundamental que o design de uma placa de circuito impresso (PCI) seja executado e fundamentado em princípios de Compatibilidade Eletromagnética (SHAHPARNIA e RAMAHI, 2004). Caso contrário, o fabricante terá problemas para obter certificação de conformidade, aumentando o custo do projeto caso está preocupação só apareça na fase de testes e produção (OTT, 2011). Ainda, de acordo com Archambeault e Dreniak (2013) o aumento da frequência de clock dos circuitos lógicos e a miniaturização das PCI amplificam fenômenos eletromagnéticos que interferem no funcionamento do próprio circuito, tais como atraso na propagação do sinal, acoplamentos, loop de corrente, emissões irradiadas, reflexões, corrente de modo comum, entre outros efeitos nocivos.

Dessa forma entende-se que profissionais que trabalhem com o design de PCI devem possuir os conhecimentos sistemáticos de compatibilidade eletromagnética e de boas práticas de projeto, os quais são aperfeiçoados continuamente através da prática profissional e da troca de experiência entre especialistas. Assim, a importância desses aspectos deve ser introduzida durante a formação do profissional, tanto em nível técnico como superior. Espera-se, dessa forma, que estratégias de aprendizagem e ensino sejam eficazes a ponto de conscientizar os discentes da importância da autonomia, da aquisição do conhecimento e da troca de experiências em sua formação profissional.

Segundo Filippin (2020), o governo brasileiro vem realizando inúmeras iniciativas desde 2000 com o objetivo de aumentar a baixa participação do setor industrial na área de semicondutores, tais como o Programa Nacional de Microeletrônica em 2002, o Programa CI Brasil em 2005, o Programa de Apoio ao Desenvolvimento Tecnológico da Industria de Semicondutores -PADIS- em 2007, entre outros. O Brasil é um grande produtor de produtos eletrônicos de consumo, e o fortalecimento de uma indústria semicondutora poderia aumentar a competividade de empresas brasileira nos mercados doméstico e internacional pois reduziria o déficit comercial devido à importação de dispositivos semicondutores (produzidos por países desenvolvidos, como França, Japão e Alemanha) na confecção de produtos eletrônicos de consumo. Logo, para que uma política pública como o Programa CI Brasil possa ocorrer com eficiência, é importantíssimo que a implementação seja feita em conjunto com universidades, indústrias e governo (FACCIN et al., 2016). 
No âmbito das universidades é imprescindível que a formação dos alunos esteja alinhada com a emancipação tecnológica do país. Em outras palavras, o papel da universidade seria tornar os discentes formados em uma mão de obra preparada e qualificada para contribuir na caminhada da indústria brasileira a níveis cada vez mais competitivos. Assim, espera-se que a disciplina proposta contribua ao fornecer as ferramentas técnicas e cultivar as habilidades humanas necessárias para formar profissionais capacitados no design de placas de circuito impresso para desempenhar um papel decisivo dentro da indústria de dispositivos eletrônicos e semicondutores.

\section{ESCOPO E PROPOSTA MULTIMETODOLÓGICA DA DISCIPLINA}

A disciplina terá como escopo o estudo, a discussão e a realização de projetos (design) de Placas de Circuito Impresso (PCI). Planeja-se seu oferecimento como uma disciplina optativa/eletiva do curso de graduação em engenharia elétrica, com sessenta horas de aula, dividida em trinta encontros de duas horas, com duas aulas por semana. Como o tema da disciplina aplica-se para praticamente qualquer área que envolva o uso de circuito integrado, os alunos serão incentivados a projetar uma PCI para aplicação em uma área específica de interesse. Essa estratégia busca incentivar o engajamento do aluno e auxiliá-lo a desenvolver suas capacidades e sua autonomia. Espera-se também que, nas diversas etapas da disciplina, o discente seja encorajado constantemente a tanto interagir com seus pares quanto a procurar o suporte necessário do professor, uma vez que tais ações promovem a extensão de suas capacidades cognitivas, seguindo o conceito da zona de desenvolvimento proximal de Vygotsky, descrita na Figura 3 (HUNT e CHALMERS, 2012).

Figura 3 - Zona de desenvolvimento proximal de Vygotsky

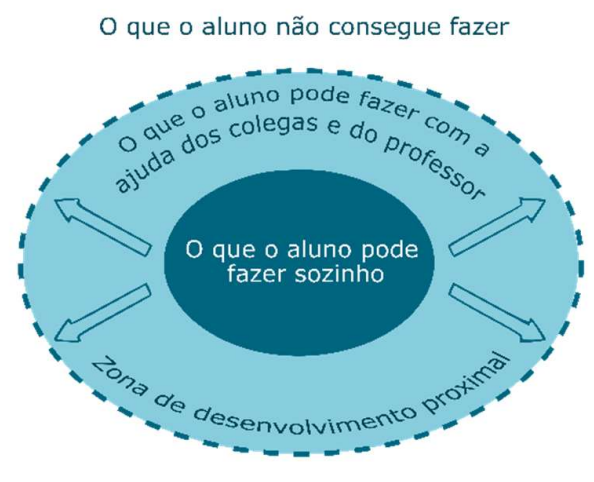

Fonte: Confeccionado pelo autor.

No aspecto metodológico, durante o semestre a disciplina incluirá três metodologias que serão divididas conforme descrito na Figura 4. Cerca de $40 \%$ do curso será ministrado com aulas expositivas e avaliações individuais, com o intuito de construir uma base sólida de entendimento da disciplina pelo aluno. Em uma segunda etapa, que consiste em $30 \%$ da disciplina, será aplicada a Metodologia Ativa de Aprendizagem Baseada em Estudo de Caso, no qual os alunos deverão avaliar e analisar projetos de PCI existentes ou já realizados. A disciplina será finalizada com uma etapa onde a Metodologia Ativa de Aprendizagem Baseada em Projetos será aplicada, na qual será proposto aos alunos que elaborem uma PCI utilizando todos os conceitos teóricos e de boas práticas estudadas durante as duas etapas anteriores. $\mathrm{O}$ detalhamento do desenvolvimento de cada etapa é descrito na seção 4.2. 
Figura 4 - Metodologias de aprendizagem utilizadas durante o semestre.

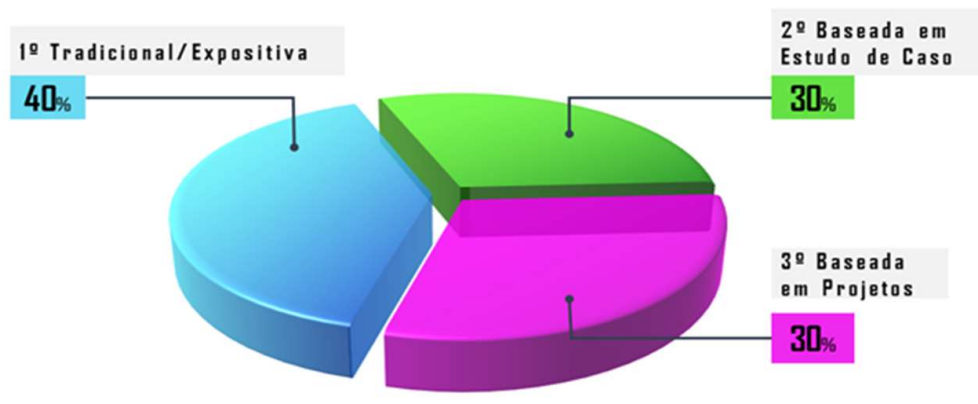

Fonte: Confeccionado pelo autor.

Em resumo, a disciplina mesclará diferentes teorias de aprendizagem, priorizando a teoria construtivista e social. A abordagem se iniciará com foco na figura do professor e, então, esse foco transitará para a figura do aluno, através da atitude do docente de pôr o aluno como protagonista no processo de aprendizagem. Tal transição deverá ser gradual para diminuir a resistência natural dos alunos de exercerem um papel de protagonismo na aquisição do conhecimento. A abordagem desta proposta multimetodológica, que mesclará a aprendizagem baseada em estudo de caso com a aprendizagem baseada em projetos, procura reconhecer as interações sociais existentes em sala de aula e alinhar as avaliações com os objetivos da disciplina. Ressalta-se que, para que o docente e o aluno possam acompanhar a evolução das atividades durante o semestre, será importante o feedback rápido, sucinto e frequente (ROSENSHINE, 2012).

\subsection{Missão do docente}

O professor tem a missão de formar profissionais capacitados para trabalhar com desenvolvimento de hardware eletrônico. Logo, a disciplina deve permitir que o aluno desenvolva as competências técnicas e sociais necessárias. No âmbito técnico, o desafio do professor é: (i) aperfeiçoar a capacidade do aluno de avaliar a melhor solução para determinado problema de acordo com os requisitos do cliente; e (ii) analisar o ambiente e as variáveis que envolvem a aplicação do produto e projetar uma PCI confiável, de baixo custo e que tenha o menor impacto ambiental possível. No âmbito social, espera-se que o profissional formado seja capaz de trabalhar em equipe, entenda o ponto de vista de terceiros, aborde os problemas de forma criativa, tenha responsabilidade, resolva conflitos, além de todas as outras competências citadas nas novas Diretrizes Curriculares Nacionais dos Curso de Graduação em Engenharia (BRASIL, 2019). Para tanto, espera-se que em sala de aula o professor tenha (i) uma postura exemplar, (ii) pontue as conquistas dos alunos como forma de aumentar a confiança e promover a autonomia, (iii) reconheça a diversidade e (iv) saiba escutar, se adaptar e reavaliar suas atitudes como docente com o objetivo de diminuir as barreiras entre aluno e professor e construir um ambiente favorável de diálogo e expressão das ideias (DAVIS, 2009).

\subsection{Estratégia}

O eixo estratégico principal da proposta multimetodológica é propor uma disciplina que avalie a caminhada do aluno na construção do conhecimento em vez de avaliar sua capacidade de reter informação. A seguir descreve-se em detalhe a proposta multimetodológica proposta.

\section{Primeira Etapa: Aulas Expositivas e Avaliação Contínua}

Os primeiros encontros da disciplina serão ministrados com aulas expositivas, cujo foco está no docente e na transmissão de conhecimento. Porém, ao longo desses encontros o professor gradualmente passará a atribuir a responsabilidade de aprender aos alunos. A avaliação nessa etapa consistirá em testes escritos realizados nos trinta minutos iniciais de cada 
encontro, cujo conteúdo serão os tópicos da aula anterior e da aula do encontro atual ("do dia"). O percentual de questões que envolve o conteúdo da aula do dia será aumentado gradativamente até que a última prova da primeira etapa seja completamente composta de assuntos que ainda não tenham sido transmitidos em sala de aula, conforme descrito na Figura 5.

Ao fim dessa etapa, o discente terá completado a fase de abordagem tradicional, que equivale a $40 \%$ do curso, e espera-se que esteja preparado para assistir as aulas voltadas aos métodos de aprendizagem ativa, aumentando a probabilidade de alcançar notas satisfatórias e ser ativo no próprio processo de aprendizagem. Em outras palavras, espera-se que o discente esteja consolidando o conhecimento gradualmente, com uma base sólida para avançar para a próxima metodologia que exige sua participação mais ativa no processo de aprendizagem.

Figura 5 - Evolução do percentual de questões relacionadas à tópicos que ainda não foram abordados em sala de aula.

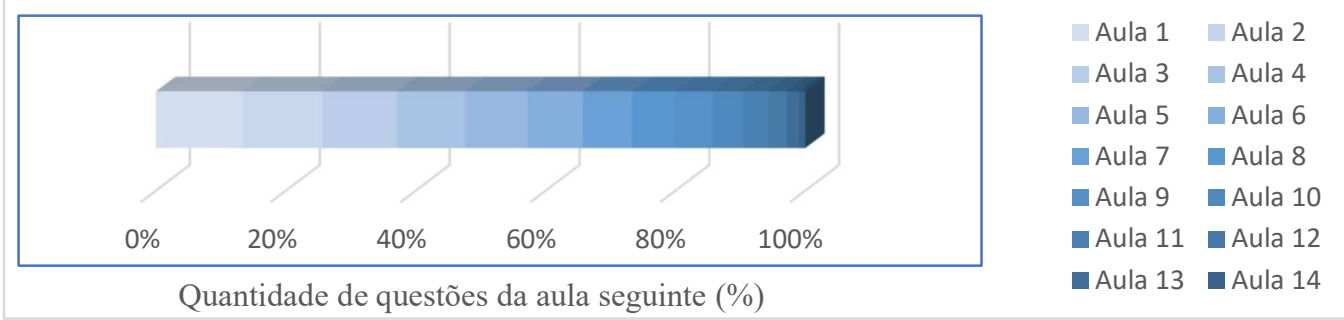

Fonte: Desenvolvido pelo autor.

\section{Segunda Etapa: Metodologia Ativa de Aprendizagem Baseada em Estudo de Caso}

Em seguida é iniciada a Metodologia Ativa de Aprendizagem Baseada em Estudo de Caso. Ao início dessa etapa (no primeiro encontro da segunda etapa ou em um momento anterior), os alunos serão divididos em grupos de quatro pessoas. $\mathrm{O}$ critério para a formação dos grupos considerará as notas da etapa anterior, sendo similar ao Método Trezentos, que prevê que alunos com desempenho melhor trabalhem com alunos com notas que não atingiram um nível satisfatório. O intuito desse critério é reduzir as diferenças de desempenho entre os grupos, além de incentivar os alunos a trabalharem com pessoas fora de seu círculo de amizade. Os grupos serão orientados a pesquisar um projeto de PCI na internet, avaliando e respondendo uma série de questões apresentadas pelo professor a cada encontro em forma de relatório. Durante os encontros, cada grupo será responsável por apresentar seu relatório para os demais colegas, descrevendo, a partir de argumentos científicos sólidos, os pontos positivos e os pontos que poderiam ser melhorados no projeto, para que os discentes possam refletir sobre as técnicas mais adequadas de design, de acordo com o escopo do projeto em análise e a função do equipamento que contém a PCI em questão. O docente atribuirá a nota com base nas apresentações e relatórios entregues.

\section{Terceira Etapa: Metodologia Ativa de Aprendizagem Baseada em Projetos}

Por fim, será aplicada a Metodologia Ativa de Aprendizagem Baseada em Projetos. Cada grupo deverá escolher uma aplicação específica para projetar uma placa de circuito impresso utilizando um software de layout de PCI de distribuição gratuita. Sugere-se que a primeira aula seja ministrada no laboratório de informática, para que os alunos assistam tutoriais para o uso do software. No encontro seguinte, os grupos terão o objetivo de criar e apresentar um cronograma de atividades, consultando o professor. Nos demais encontros, cada grupo fará apresentações acerca do cumprimento das tarefas propostas e das decisões tomadas durante as atividades do projeto. Nesta etapa, as avaliações serão feitas com base nesses aspectos e nas discussões levantadas a cada apresentação. O objetivo é que todos possam dar sugestões de melhoria e entendam melhor as soluções e boas práticas adotadas por cada grupo. 


\section{Objetivos e Avaliações de cada Etapa}

A Tabela 1 apresenta a avaliação que será aplicada e os respectivos objetivos esperados em cada etapa da disciplina, sendo estruturados a partir da Taxonomia de Bloom. Observa-se que as avaliações terão correções rápidas e sucintas para que cada discente mantenha sua dedicação semanal e assim seja capaz de regular de forma autônoma sua aprendizagem. As correções rápidas também trarão um feedback ágil ao professor, permitindo-o a adequar sua postura e ajustar a metodologia atual de acordo com o desempenho dos alunos. Assim, espera-se que os alunos sejam avaliados da melhor maneira possível, evitando inconsistências nas notas.

Tabela 1 - Avaliações alinhadas com os objetivos educacionais de acordo com a metodologia aplicada.

\begin{tabular}{|c|c|c|}
\hline Metodologias & $\begin{array}{c}\text { Objetivos } \\
\text { Educacionais }\end{array}$ & Avaliações \\
\hline Trac & $\begin{array}{ll}\text { - } & \text { Resolver } \\
\text { - } & \text { Pesquisar } \\
\text { - } & \text { Recordar } \\
\end{array}$ & Pro \\
\hline $\begin{array}{c}\text { Aprendizagem } \\
\text { Baseada em Estudo } \\
\text { de Caso }\end{array}$ & $\begin{array}{ll}\text { - } & \text { Compreender } \\
\text { - Avaliar } \\
\text { - Analisar }\end{array}$ & $\begin{array}{l}\text { Apresentação e } \\
\text { Relatório }\end{array}$ \\
\hline $\begin{array}{c}\text { Aprendizagem } \\
\text { Baseada em Projetos }\end{array}$ & $\begin{array}{ll}\text { - } & \text { Aplicar } \\
\text { - } & \text { Desenvolver } \\
\text { - } & \text { Justificar }\end{array}$ & $\begin{array}{l}\text { Apresentação } \\
\text { e Projeto }\end{array}$ \\
\hline
\end{tabular}

Fonte: Desenvolvido pelo autor.

\section{CONSIDERAÇÕES FINAIS}

Aplicar uma disciplina com três diferentes metodologias não é uma tarefa trivial. Caso essa proposta multimetodológica seja aplicada sem uma experiência prévia ou sem o conhecimento das metodologias adotadas, o professor pode prejudicar o processo de aprendizagem dos discentes. Ressalta-se que a proposta multimetodológica pode exigir maior preparo de aulas que o esperado de uma disciplina nos moldes tradicionais. Assim, recomenda-se fortemente que o docente, caso não tenha experiência com metodologias ativas de aprendizagem, aplique a proposta multimetodológica de forma gradual ao longo de vários semestres. Por exemplo, apenas a primeira etapa seria aplicada no primeiro semestre, adicionando as demais etapas gradualmente no decorrer dos próximos semestres, de acordo com o desenvolvimento das habilidades do docente.

Dentro das possibilidades do contexto da disciplina apresentada neste artigo, sugere-se a criação de um repositório público na plataforma GitHub com todos os trabalhos que seriam produzidos pelos alunos, com o intuito de construir e disseminar conhecimento científico e técnico para a comunidade interessada em PCIs e desenvolvimento de hardware eletrônico. $\mathrm{O}$ uso do GitHub também permite ao professor averiguar se algum grupo está utilizando algum projeto indexado anteriormente no repositório. Caso isso se confirme, sugere-se que os discentes sejam orientados apenas a refazer o projeto e apresentá-lo novamente. Porém, é recomendado que o discente tente identificar o motivo que levou o grupo a tal prática, tomando uma postura amigável e compreensiva. Caso exista alguma variável na metodologia que favoreça esse tipo de atitude, cabe ao professor readequar a metodologia.

Apesar da disciplina em discussão ser relacionada ao design de PCI, a proposta multimetodológica pode ser aplicada para qualquer disciplina que englobe em seu escopo o desenvolvimento e análise de projetos. 
"Os desafios para formar hoje o engenheiro do amanhã"

\section{Agradecimentos}

O presente trabalho foi realizado com apoio da Coordenação de Aperfeiçoamento de Pessoal de Nível Superior - Brasil (CAPES) - Código de Financiamento 001.

\section{REFERÊNCIAS}

ARCHAMBEAULT, Bruce R.; DREWNIAK, James. PCB design for real-world EMI control. Springer Science \& Business Media, 2013.

BEHRENS, Marilda. O paradigma da complexidade na formação e no desenvolvimento profissional de professores universitários. Educação, v. 30, n. 63, p. 439-455, 2007.

BIGGS, John B. Teaching for quality learning at university: What the student does. McGraw-hill education (UK), 2011.

BIGGS, John. Enhancing teaching through constructive alignment. Higher education, v. 32, n. 3, p. 347-364, 1996.

BLOOM, Benjamin S. et al. Taxonomy of educational objectives. Vol. 1: Cognitive domain. New York: McKay, p. 20-24, 1956.

BRASIL. Diretrizes Curriculares Nacionais do Curso de Graduação em Engenharia. [S. 1.], Ministério da Educação e Conselho Nacional da Educação, 23 jan. 2019. Disponível em: http://portal.mec.gov.br/index.php?option=com_docman\&view=download\&alias=109871-pces00119-1\&category_slug=marco-2019-pdf\&Itemid=30192. Acesso em: 21 set. 2020.

BRYSON, Colin; HAND, Len. The role of engagement in inspiring teaching and learning. Innovations in education and teaching international, v. 44, n. 4, p. 349-362, 2007.

DAVIS, Barbara Gross. Tools for teaching. John Wiley \& Sons, 2009.

FACCIN, Kadígia; BORTOLASO, Ingridi; BALESTRIN, Alsones. A visão relacional de políticas de ciência e tecnologia: o caso do Programa CI Brasil. REAd. Revista Eletrônica de Administração (Porto Alegre), v. 22, n. 1, p. 226-251, 2016.

FERRAZ, Ana Paula do Carmo Marcheti; BELHOT, Renato Vairo. Taxonomia de Bloom: revisão teórica e apresentação das adequações do instrumento para definição de objetivos instrucionais. Gestão \& Produção, v. 17, n. 2, p. 421-431, 2010.

FILIPPIN, Flavia. Estado e Desenvolvimento: a indústria de semicondutores no Brasil. 2016. 266p. Dissertação (mestrado) - Universidade Estadual de Campinas, Instituto de Economia, Campinas, SP.

FRAGELLI, Ricardo Ramos; FRAGELLI, Thaís Branquinho Oliveira. Trezentos: a dimensão humana do método. Educar em Revista, n. 63, p. 253-265, 2017.

GARAVALIA, L., HUMMEL, J., WILEY, L., \& HUITT, W. Constructing the course syllabus: Faculty and student perceptions of important syllabus components. Journal of Excellence in College Teaching,10(1), 5-22, 1999.

GUEDES, Leonardo Guerra de Rezende; SANTOS, João Pedro Aguiar dos. Diagnóstico e avaliação da aplicação de metodologias ativas nos cursos de engenharia da Pontifícia Universidade Católica de Goiás e da Universidade Federal de Goiás. Brazilian Journal of Development, v. 5, n. 9, p. 16897-16910, 2019.

HUNT, Lynne; CHALMERS, Denise. University teaching in focus: A learning-centred approach. Routledge, 2012.

KRATHWOHL, David R.; ANDERSON, Lorin W. A taxonomy for learning, teaching, and assessing: A revision of Bloom's taxonomy of educational objectives. Longman, 2009. 
LEFRANCOIS, Guy R. Theories of human learning. Cambridge University Press, 2019.

MENEGHEL, Stela M.; ROBL, Fabiane; SILVA, Tattiana T. A relação entre avaliação e regulação na educação superior: elementos para o debate. Educar em Revista, n. 28, p. 89-106, 2006.

OTT, Henry W. Electromagnetic compatibility engineering. John Wiley \& Sons, 2011.

PAUL, Clayton R. Introduction to electromagnetic compatibility. John Wiley \& Sons, 2006.

PRETTO, Nelson De Luca; RICCIO, Nicia Cristina Rocha. A formação continuada de professores universitários e as tecnologias digitais. Educar em Revista, n. 37, p. 153-169, 2010.

PRINCE, Michael; FELDER, Richard. The many faces of inductive teaching and learning. Journal of college science teaching, v. 36, n. 5, p. 14, 2007.

ROSENSHINE, Barak. Principles of Instruction: Research-Based Strategies That All Teachers Should Know. American educator, v. 36, n. 1, p. 12, 2012.

SHAHPARNIA, Shahrooz; RAMAHI, Omar M. Electromagnetic interference (EMI) reduction from printed circuit boards (PCB) using electromagnetic bandgap structures. IEEE Transactions on Electromagnetic Compatibility, v. 46, n. 4, p. 580-587, 2004.

SOUZA, Pricila Rodrigues de. Aprendizagem significativa e alinhamento construtivo uma proposta para o ensino de circuitos elétricos. 2016. 149p. Dissertação (mestrado profissional) - Instituto Federal do Amazonas (IFAM), Campus Manaus Centro, Manaus, AM.

TENNANT, Mark; MCMULLEN, Cathi; KACZYNSKI, Dan. Teaching, learning and research in higher education: A critical approach. Routledge, 2009.

\section{A MULTI-METHODOLOGICAL PRACTICE FOR TEACHING A PRINTED-CIRCUIT BOARD DESIGN CLASSES}

Abstract: This article presents a multi-methodological practice to cope with resistance of students when engaging in active learning classes. Specifically, it is aimed for an optional course, which includes the study, analysis and design of Printed Circuit Boards (PCBs), in an undergrad Electrical Engineering Course. PCB-design practitioners and engineers must (i) be active on improving their knowledge, (ii) be able to discuss complex issues on PCB design with costumers and colleagues and (iii) be comfortable in a teamwork environment, which leads to a need to start fostering such abilities in university courses. Considering aspects of the Biggs' Constructive Alignment and Bloom's Taxonomy, one may consider that fostering cognitive abilities that goes beyond the simple acquisition of data is founded on improving teacher-student relationship and building a hierarchical framework of student's core capabilities through the course. Teachers have been using active methodologies on a mission to help students develop skills in addition to those promoted by traditional behavioral methods. Problem-based learning (PBL) is an excellent choice to the proposed course. Nevertheless, interpersonal relationship issues and the self-learning framework faced by students are the main barriers to the effective implementation of PBL. The multi-methodological practice applied to a PCB-related course is then described in detail, emphasizing that evaluation system changes along it accordingly to the current methodological aim to facilitate the adaptation of students and to increase their engagement in the course. The social and constructive approach of the multi-methodological proposal allows its application to any class that involves design and project features.

Keywords: Biggs' Constructive Alignment. Bloom's Taxonomy. Electromagnetic Compatibility. PCB design. Teaching in Engineering. 